\title{
Algunos comentarios a la operación de Manchester - Fothergill
}

\author{
Por Ricardo Rueda González.
}

Miembro afiliado de la S. C. O. G. - Interno de Clínica Ginecológica del Hospital San Juan de Dios de Bogotá.

La operación de Manchester-Fothergill es uno cie los procedimientos quirúrgicos que han obtenido más aceptación en muchos de nuestros medios para la corrección del prolapso genital y a la que se han introducido varias modificariones y se han agregado algunos artificios que han dado resultados bastanic satisfactorios en manos de sus seguidores.

Donal, de Manchester, en el año 1888 (1) fue el primero que trató el prolapso uterino mediante ia colporrafía anterior y posterior acompañada de la amputación del cuello uterino por el método de Shroder. Posteriormente Fothergill y Shaw, colaboradores del anterior, introdujeron algunas modificaciones como son ia disección extensa de la pared vaginal anterior y la interposición de la base de los ligamentos anchos sobre la cara anterior del muñón cervical. Así quedó constituída la operación para la cura del prolapso genital denominada de Manchester-Fothergill.

Nosotros empleamos esta técnica de corrección del prolapso genital teniendo en cuenta varios factores.

a) El grado del prolapso.- La realizamos para cura de histeroceles de primero y segundo grados, acompañados de recto o cistocele, ya que si estos están presentes, pueden también corregirse sin alguna dificultad, y en los de tercer grado siempre que no existan concomitantemente entero, recto o cistoceles demasiado grandes.

b) La edad de la enferma.-Por regla general podemos decir que sometemos a este procedimiento a las mujeres con prolapso genital, no mayores de cuarenta años. 
c) Porvenir obstétrica.-Dado que wí es posible ei embarazo en la mujer que ha sido sometida a este tipo de operación, es la de elección en la mujer que desea continuar su función reproductora.

d) Ginecopatías concomitantes.-En lo que respecta a este factor, podemos decir lo siguiente: si la ginecopatía se puede corregir en el mismo acto operatorio, mediante alguna modificación de ia técnica o agregándole algusos tiempos, como puede ser, por ejempio, la apertura del fondo de saco vésico uterino para extirpar una trompa enferma o un pequeño blastoma ovárico, la practicamos. Si por el contrario, la ginecopatía no puede ser tratada por estos procederes, ya por presentar una gran dificuitad técnica o por tratarse de un proceso maligno, abandonamos el sistema y hacemos uso de otros. De aquí ia importancia fundamental que tiene la práctica del legrado biopsia del endometrio antes de proccder a realizar la cura del prolapso genital, que muy pocos hacen, especialmeste en el medio hospitalario, y que representa todas las ventajas.

e) Estado general de la enferma.-En pacientes en mal estado general, se puede hacer la operación de Manchester-Fothergill ya que es la más sencilla, la más breve y la que da lugar a estados da shock menos intensos.

Habiendo analizado ya estos factores podemos decir que la operación tiene las siguientes ventajas: conserva un puente de tejido vivo y fuerte de un ligamento ancho al otro a través diel muñón del cuello y del cuerpo uterino. La irrigación de las partes que restan quedan ampliamente conservada, evitándose en esta forma la necrosis de los tejidos que se desearon respetar durante el acto operatorio. La posibilidad de recidiva de ia bóveda vaginal y de la pared vaginal anterior quedan reducidas. Las posibilidades de embarazo no son despreciables, siendo posible que las pacientes lleven el embarazo a término y den a luz por ía vía vaginal sin complicación alguna si se practican ciertas modificaciones a la técnica que más adelante anotaremos. Cuando se practica la operación tal cual es saivo accidentes, no se entra en la cavidad peritoneal; por lo tanto, cualquier hemorragia postoperatoria o reacción inflamatoria muy seguramente sean extraperitoneaies y drenen hacia afuera, en lugar de difundirse hacia la cavidad peritoneal, hecho que constituye una de las ventajas sobre la histerectomía vaginal. 
Ya habíamos anotado que a la operación en estudio se le pueden hacer algunas modificaciones y aumentarle algunos tiempos con el fin de corregir o tratar afecciones concomitantes. Queremos mencionar a continuación ias que se han hecho en el Servicio de Ginecología del Hospital San Juan de Dios, de Bogotá y las que hemos visto practicar a algunos ginecólogos de otros centros.

Algunos practican la incisión clásica, sobre la pared vaginal anterior, que se extiende desde unos pocos milimetros por debajo del meato urinario hasta la unión de dicha pared con el cuello uterino por la ínea media. Otros, siguiendo a Shaw (2), extirpan un segmento oval de la mucosa para no tener que seccionar posteriormente los colgajos restantes de la misma. Finalmente, otros extirpan un colgajo romboide, cuyos dos extremos más distantes se encuentran en el reborde inferior del meato urinario y en el cuello uterino, respectivamente, y sus dos extremos más próximos en puntos equidistantes de ía ínea media, según la cantidad de mucosa que se desee resecar.

Nos parece más práctico y más fácil para establecer posteriormente el calibre vaginal, emplear el procedimiento clásico en este tiempo de la intervención.

El tratamiento que se le da a la aponeurosis vesicovaginal varia también de unas manos a otras. Entre nosotros los doctores Jorge Amorocho y Gonzalo Echeverri P. (3), separan ampliamente la mucosa vaginal de la aponeurosis, formando una especie de alero con el colgajo de ésta, y sostienen que la reconstrucción del piso vesical será más firme. Disecando conjuntamente la mucosa vaginal y la aponeurosis vesico-vaginal, otros (4) prefieren hacer la reconstrucción del piso vesical pasando por alto el tiempo anterior que es a veces proiongado y no exento del percance de seccionar en algunos trayectos la mucosa vaginal que posteriormente ha de ser seccionada, y que por esta razón no ofrece peligro alguno.

Hay otro tiempo de la técnica que hemos visto hacer de diversas maneras, y es el tratamiento de los ligamentos de Mackenrrodt. Lo clásico es su sección a ras del istmo uterino y luego su sutura, previo entrecruzamiento, sobre la cara anterior del muñón cervical. Pero como ya anotamos se les puede dar diversos tratamientos; cuando están demasiado flácidos los hemos visto acortar mediante la sección de alguna porción o mediante un entrecruzamiento forzado. También los hemos visto entrecruzar sobre el sitio ordinario sin seccionarlos previamente. 
Nos parece más aceptable seguir el procedimiento clásico, ya que mediante la sección previa al entrecruzamiento y sutura sé puede hacer una amputación más alta del cuello cuando se necesita. Los defensores del entrecruzamiento sin sección dicen que en esta forma se evita seccionar los vasos cervicales que se encuentran hacia la parte interna del ligamento, asegurando así la vitalidad del mismo.

La extensión de la amputación cervical no es un detalle despreciable, ya que de este tiempo puede depender el porvenir obstétrico de la enferma, y deberá variar en cada caso particular.

En efecto, cuando existe alguna lesión macro o microscópica del cuello como puede ser, por ejemplo, la presencia de un cuello hipertrófico, la hipertrofia de aiguno de sus labios, la concomitancia del prolapso con una cervicitis crónica, laceración o inclusive un carcinoma "in situ" para no citar sino unas pocas, se requiere una amputación bastante extensa. De lo contrario, en presencia de un cuello sano, pequeño y correctamente epitelizado, se deberá limitar al máximo la ampuíación, especialmente si la enferma se encuentra en una edad que no pase de los treinta y ocho o cuarenta años.

El Jefe del Servicio de Ginecología de este hospital, profesor Arturo Aparicio (5), es uno de los principales partidarios de esta conducta, y cuenta con numerosos casos de embarazo posterior a la operación comentada, en los cuales no se presentó distocia alguna al momento del parto.

La reconstrucción del conducto cervical y la cubierta de la superficie cruenta del muñón cervical, la practicamos siempre mediante la colocación de Puntos de strumdorf, que no son otra cosa que puntos de colchonero que invaginan el borde cruento de la mucosa vaginal dentro del canal cervical. Generalmente, y siguiendo a los clásicos, coiocamos dos de estos puntos, anterior y posterior, dejando para las partes laterales unos pocos puntos en $\mathrm{X}$ que completan el afrontamiento de las superficies cruentas. Sin embargo el profesor Aparicio coloca también en las partes laterales los puntos de Strumdorf, consiguiendo en esta forma una invaginación más completa. Problema que se presenta con frecuencia después de la traqueloplastia es la estenosis del conducto cervical, complicación que se puede evitar por alguno de los procedimientos que hemos empleado con resultados satisfactorios. 
Uno de ellos es hacer una buena dilatación cervical antes de empezar la operación para practicar el legrado biopsia, y colocar una vez terminada un tallo de Iribarne o una sonda de caucho fuerte. Otro es hacer la dilatación mediante el diiatador de Goodell o cualquier otro modelo similar, antes y después de la intervención, sin dejar tallo o sonda alguna. Los seguidores de uno u otro procedimiento dicen obtener buenos resuitados, pero no sobra decir que es siempre prudente hacer un control del conducto cervical antes dei décimoquinto día del postoperatorio, con el fin de practicar algunas dilataciones si se ha presentado la estenosis.

De los tiempos que se pueden agregar a ia operación de Manchester-Fothergill, figura entre los más importantes la corrección de la retroversión uterina, y para ello se puede hacer la técnica de Baldy-Webster a través de una apertura practicada a nivel de fondo de saco vésico-uterino. Aparicio sostiene que se puede practicar sin alguna dificultad y se la hemos visto hacer con todo éxito, descartando en esta forma una desventaja que presenta la operación de Manchester que pudiéramos llamar pura.

No quiero terminar este comentario sin hacer algunas consideraciones respecto al material de sutura empleado y a los cuidados pre y post-operatorios por seguir.

Hemos conseguido buenos resultados mediante el empleo del catgut crónico número 1 para la unión de la aponeurosis vésicovaginal, materiai que posee una fuerza tensil suficiente para el efecto buscado; sin embargo, a falta de éste usamos igualmente el número 0 con resuitados similares. De la sutura de los ligamentos cardinales podemos decir algo parecido. La mucosa vaginal la suturamos indistintamente con catgut simple o cromado, 0 o 1 , con puntos separados o continuos.

En los cuidados post-operatorios inmediatos seguimos esta rutina:

Un taponamiento de gasa embebida en ace:te estéril con una c dos tabletas vaginaies de Terramicina de 100 mgs., que al mismo tiempo que hace la desinfección del medio vaginal, elimina cualquier espacio muerto que haya persistido. La retiramos a las 24 horas. Colocación en la vejiga de una sonda del tipo Foley o Pezzer que retiramos a las 48 o 72 horas salvo complicaciones. Si se practica uretroplastia preferimos el tipo Foley, ya que si se retirara no causa traumatismo aiguno sobre la uretra. 
En cuanto a la movilización de las enfermas, la tendencia actuai es la de levantarlas después de las 48 horas, particularmente si son de edad. Los resultados han sido satisfactorios y no hemos observado recidiva de la sintomatología local con este proceder.

\section{S U M A R I O}

1) Se hace una breve reseña histórica de ia operación de Manchester-Fothergill.

2) Se anotan los factores que se tienen en cuenta para realizar la operación.

3) Se hacen resaltar las ventajas de la técnica sobre otras empleadas para la cura del prolapso genital.

4) Se describen las modificaciones a la técnica habitual y los tiempos que pueden agregarse para corregir algunas ginecopatías concomitantes, y se hacen algunos comentarios a ellas.

5) Se cuenta la experiencia tenida con algunos materiales de sutura.

6) Se exponen los cuidados post-operatorios inmediatos de rutina.

\section{BIBLIOGRAFIA}

1. The Linde, Richard.- "Ginecología Operatoria". 1 Ed. Española. Buenos Alres. 1948. Ed. José Bernardes. M

2. Aaorn E. Kanter and Arthur H. Klawas.- "Prolapse of the uterus. An operation for its relief". Journal of In. Col. of Sr. XVI-I July, 1951.

3. Amorocho Jorfe y Echeverri Gonzalo. Referencia personal. 1954.

4. Cómez T. Alberto.- - "Demostraciones prácticas al curso de Ginecología. Hospital San Juan de Dios. Bogotá. 1954.

5. Aparicio J. Arturo.- "Seminarios de esterilidad. Reunidos en el Hospital San José de Bogotá. 1953. 